\title{
Discussion on Mental Health Problems Related to COVID-19 for College Students in China
}

\author{
Shuyu Rao
}

Jiangsu Meicun Senior High School, Jiangsu, 214112, China

*Corresponding author. Email: oohrena@163.com

\begin{abstract}
Mental health problems are common and these problem share a greatly influence on people's thinking, mood, and behavior. Mental health problems can be prevented and recovered by intervention and treatments. Chinese college students are reported to be vulnerable to psychological problems. Since COVID-19 (Coronavirus Disease 2019) broke out, studies found that students have been experiencing higher levels of mental health problems including anxiety, stress, and depression with a high incidence rate. This article reviews and analyzes the potential risk factors reported in various studies including the vulnerable populations (people who encounter the infection of their families, friends or other relatives, women and people with prior mental health problems), more extensive attention paid to media, lower perceived support from society and medical students were reviewed and analyzed. Then the corresponding solutions were discussed. Government and schools should pay more special attention to those typically vulnerable populations, release official updates at regular intervals, help students to aware of the effects of complicated information online and reduce the time directly focused on social media, and provide more social support to those students; sufficient training on dealing with COVID-19 and regular supervision on those students' health should be added for medical students. The specific psychological intervention strategies for prevention and surveillance of Chinese college students' psychological health will get better in the future.
\end{abstract}

Keywords: College Student, COVID-19, Mental Health Problems, Vulnerable Population

\section{INTRODUCTION}

As corona virus continues globally, mental health symptoms associated with COVID-19 have been common for the general population [1]. Mental health meaning emotional, psychological, and social well-being, is just like physical health and is important at each period of life. Good mental health leads to living a positive life, while poor mental health may impair abilities of thinking, feeling or reacting. Mental health problems can be prevented, treated and surveilled by developing different methods according to different causes.

In China, the students' mental health is getting more concerned than before. Most current college students have little problem solving abilities and social communication skills before entering college because of the education system focusing on examinations and the policy of family planning ( "One-chind" Policy) in China. As a consequence, college students in China are reported to be vulnerable to depression [2], especially during the outbreak of COVID-19 [3,4]. According to studies, various mental health problems (including anxiety, depression, suicide etc.) have more and more critically impacted college students since the outbreak of COVID-19. Identification, treatment, and follow-up for mental health problems among college students in real life are difficult because they are unwilling to seek help. So the specific psychological intervention strategies for Chinese college students should be designed. Therefore, this article aims to analyze the risk factors that could induce those mental health problems and how to effectively deal with them. First, the author would discuss the condition of mental health problems related to COVID-19 for college students. Then, risk factors that may cause these problems would be analyzed according to recent studies and reality. In the end, targeted solutions will be discussed. 


\section{EPIDEMIOLOGY OF MENTAL HEALTH PROBLEMS}

\subsection{Overview of Mental Health Problems}

Mental health including emotional, psychological and social well-being has a great impact on people' $\mathrm{s}$ thoughts, emotions and behavior. Mental health problems are affected by many factors, which includes biological factors, prior experiences, genetic factor, and life way, etc. Poor mental health might result in difficult thinking, feeling or reacting as usual, which is as bad as a physical illness, or even worse. Psychological problems vary in type, including anxiety disorders, behavioral disorders, emotional disorders, suicide, trauma and stress-related disorders, etc. Some of them have similar symptoms. Conditions of patients with mental health problems can be improved and many problems might be fully cured if he or she get help such as talk therapy and/or medicines.

Widespread outbreaks of COVID-19 have a great adverse influence on both mental and physical health among the general population. Several studies suggest that people have various types of mental health problems, including depression, anxiety disorders, stress, panic, anger, impulsivity, somatization disorder, sleep disorders, emotional disturbance, post-traumatic stress disorder, suicidal behavior, and so on during the epidemic of COVID-19. The prevalence of mental health problems differs remarkably from population to population.

\subsection{Prevalence of Chinese College Student}

During the epidemic of COVID-19, Chinese college students had to take courses through web-based applications or other teaching activities. Thus, the uncertainty for academic progression might have negatively affected college students' mental health. College students, studies and uncertain future employment have been negatively affected by COVID-19. The effect results in college students' anxiety. Mental stress has also become more severe because of the quarantine during COVID-19, which has made the relationships between people to be less close. Previous studies showed that mental problems or mental illnesses in different countries during the outbreak of COVID-19 were quite common among college students. One study from China demonstrated that about $45 \%$ of 746217 college students suffered mental health problems and the prevalence rates of mental health problems (acute stress: 34.9\%, depression:21.1\%, anxiety: $11.0 \%$, respectively) were high [3]. And another study from France depicted that about $42.8 \%$ of the participants had at least one kind of mental health problem (self-reported suicidal thoughts, severe distress, stress, anxiety, and depression) in 69054 college students [5]. A meta-analysis showed the pooled prevalence rate of depressive symptoms in college students in China was 26.0\% (95\%CI: 23.3-28.9\%) during the pandemic of COVID-19 [6]. Furthermore, the prevalence of anxiety disorders and depression among college students was affected by the geographic location of the college [7].

\section{POTENTIAL RISK FACTORS}

Realizing potential risk factors of college students' mental health problems is the main step toward finding the corresponding solutions. Several studies demonstrated that there are some epidemic and psychosocial risk factors for people with mental health problems including people who encounter the infection of their families, friends or other relatives, frequent social media use, low perceived social support, women, senior year and history of mental health problems.

\subsection{Vulnerable Populations}

The vulnerable populations include people who encounter the infection of their families, friends or other relatives, women and people with prior mental health problems.

Studies showed that family members and friends being infected by COVID-19 is remarkably correlated with a high risk of probable acute stress, depressive and anxious symptoms among college students $[3,8]$. This might be due to fear of infection for the high infectivity of the COVID-19. Moreover, the college students may experience hardship for struggling with COVID-19 and even death from families/relatives or their friends, and even they might also suffer more mental distress.

Many studies have considered women to be a significant risk factor related to mental health problems. Women were reported to be easier to suffer depression, anxious emotion and the symptoms of insomnia etc in comparison with men. The possible explanations might be that women are less likely to conduct problem-solving strategies effectively, and were thought to be more sensitive to stress hormones.

Furthermore, students in the senior year were at higher risk of being anxious. Obviously, the impact of academic delays on the anxiety symptoms of senior students is greater than that of freshmen. Furthermore, worrying about future employment is also a potential risk factor that results in anxiety. Studies showed that students with prior mental health problems, who have lower levels of psychological resilience, are remarkably associated with higher level of anxious and depressive symptoms. 


\subsection{Prolonged Media Exposure}

Social media on the internet is one of the main channels for college students to get and update the COVID-19 information. Studies showed that prolonged media exposure of the COVID-19 was a strong risk factor for probable acute stress and anxiety symptoms. Ma et al[3] reported that students' media exposure of the COVID-19 $\geqslant 3 \mathrm{~h} /$ day were $>2$ times more likely to have probable acute stress than those who were exposed to $<1 \mathrm{~h} /$ day. There are mainly two explanations for the information above. Firstly, it is difficult for college students to search credible and useful information with excessive conflicting information on internet, so massive media coverage may provide a way for receiving negative information leading to probable acute stress and anxiety symptoms. Moreover, many people delivered their bad feelings, such as fear, worry, nervousness, anxiety et al. on social media. Repeatedly media content related to trauma may extend acute stress experiences and lead to abundant stress-related symptoms [9].

The timely release of accurate and updated health information related to COVID-19 by health authorities was reported to have a relationship with less possibility of anxiety, stress, and depression [10].

\subsection{Low Perceived Social Support}

Perceived social support, a key source of emotional support, was correlated with a lower probability of anxiety and depressive symptoms [3,8,11]. Social interactions can reduce negative emotions such as anxiety and can improve mood and also may contribute to improving self-efficacy and leading to more encouragement. Cao et al[8] demonstrated that social support was adversely associated with the anxiety of college students. As a result, psychosocial support from family, friends, and schools plays an important role for college students' physical and mental health during epidemic of the COVID-19. The established online psychological counseling services (eg, WeChat-based resources) and psychological assistance hot-lines in hospitals, universities, and academic societies as well as providing psychological self-help intervention systems for psychological crises intervention are helpful for college students [12].

\subsection{Medical Students}

Medical education often involves practice and experiments. However, policies related to COVID-19 changed the way of medical courses, especially for the loss of the opportunity for clinical training at the bedside and exposure to high-risk environments which may lead to high risk of developing worry, anxiety, or even depression among medical students. On the other hand, the medical students especially for 4th or 5th year undergraduates acquire more knowledge of clinical medicine and have a higher knowledge level of COVID-19, which may result in high self-efficacy and self-esteem. One study from Turkish showed that clinically significant anxiety prevalence was $23.2 \%$ [13]. A large-scale study with 5,982 medical students from China demonstrated that poor psychological statuses including mild to severe depressive symptoms $(35.2 \%)$ and anxiety symptoms (22.8\%) were prevalent. Low social support for college students was demonstrated to be a stronger risk factor related to poor mental health while probable exposure and regional outbreak for COVID-19 were weaker risks. Furthermore, 1st to 3rd year undergraduates had higher anxiety symptom possibilities compared with 4 th or 5 th year undergraduates [14]. Another study from China reported that the medical students had fewer moderate to severe anxiety and stress symptoms, a higher level of knowledge for COVID-19, and a higher state of consciousness in comparison with the non-medical students [15].

\section{DISCUSSIONS ON TARGETED SOLUTIONS}

According to the four aspects of risk factors, the author discusses certain solutions that might remit the current situation of college students during the COVID-19.

\subsection{Vulnerable Population}

Health authorities need to identify highly vulnerable among the college students, then specific attention should be paid to the above-mentioned high-risk populations for early psychological interventions. The factors discussed should be taken into consideration for effective psychosocial interventions by schools and governments, aiming to positive mental health results and psychosocial resilience.

\subsection{Prolonged Media Exposure}

It is important for the government to release official updates at regular intervals which needs to be based on evidence and to monitor social media to reduce the negative information. Additional information updating on the number of infected cases and location were important for reducing levels of anxiety. Moreover, college students should be aware of this effect and try to get the information through official channels.

\subsection{Low Perceived Social Support}

The helpful psychosocial supports should include helping students feel safe, teaching the students on basic knowledge of COVID-19 especially for how to protect 
themselves; helping students learn the ways to get trustworthy information and to ask for help; creating the conditions for appropriate activities in school and getting in contact with family and friends during a movement restriction period; providing measures and suggestions on how to get relaxed and offering calming techniques using various media.

\subsection{Medical Students}

In order to improve and even ensure the mental health of medical students, high-level social support is supposed to be highlighted for the high-risk medical students such as female gender and 1st to 3rd year undergraduates etc. Colleges or universities can offer students appropriate supervision and full training on COVID-19 to assure students' safety when the students work as an intern in the teaching hospitals. In addition, learning self-help methods and participating in some mental health education programs might give the situation some help because the anxiety and depression among the medical students were relatively mild. Furthermore, resilience training can help students to become more confident and more competent when facing challenges on health emergencies in the future.

\section{CONCLUSIONS}

During the outbreak of COVID-19 in China, mental health problems become relatively high among college students in China. In the article, the author described the situation of college students' mental health problems during COVID-19, analyzed the potential risk factors, and offered certain solutions. According to published studies and the analysis above, the author found that the risk factors mainly about vulnerable populations, prolonged media exposure, low perceived social support, and medical students. To avoid these potential risk factors and thus release the psychological problems among college students, the author offered certain advice. Government and schools should pay more special attention to those typical vulnerable populations, release official updates at regular intervals, help students to be aware the effects of complicated information online and reduce the time directly focused on social media, and provide more social support to those students. For those medical students, sufficient training on dealing with COVID-19 and regular supervision on those students' health are also necessary. This might be helpful for reinforcing prevention and surveillance for the mental health of college students in the future development, and developing appropriate mental healthcare strategies for college students in China.

\section{REFERENCES}

[1]. Shi L, Lu ZA, Que JY, et al.Prevalence of and Risk Factors Associated With Mental Health Symptoms
Among the General Population in China During the Coronavirus Disease 2019 Pandemic. JAMA Netw Open. 2020;3(7):e2014053. doi: 10.1001/jamanetworkopen.2020.14053.

[2] Gao L, Xie Y, Jia C, Wang W.Prevalence of depression among Chinese university students: a systematic review and meta-analysis. Sci Rep. 2020;10(1):15897. doi: 10.1038/s41598-020-72998-1.

[3] Ma Z, Zhao J, Li Y, et al. Mental health problems and correlates among 746217 college students during the coronavirus disease 2019 outbreak in China, Epidemiol Psychiatr Sci. 2020,29:e181. doi: 10.1017/S2045796020000931.

[4] Luo W, Zhong BL, Chiu HF. Prevalence of depressive symptoms among Chinese university students amid the COVID-19 pandemic: a systematic review and meta-analysis.Epidemiol Psychiatr Sci. 2021;30:e31. doi: $10.1017 /$ S2045796021000202.

[5] Wathelet M, Duhem S, Vaiva G, et al. Factors Associated With Mental Health Disorders Among University Students in France Confined During the COVID-19 Pandemic, JAMA Netw Open. 2020,3(10):e2025591. doi: 10.1001/jamanetworkopen.2020.25591.

[6] Luo W, Zhong BL, Chiu HF.Prevalence of depressive symptoms among Chinese university students amid the COVID-19 pandemic: a systematic review and meta-analysis.Epidemiol Psychiatr Sci. 2021;30:e31. doi: $10.1017 /$ S2045796021000202.

[7] Wu X, Tao S, Zhang Y, et al.Geographic Distribution of Mental Health Problems Among Chinese College Students During the COVID-19 Pandemic: Nationwide, Web-Based Survey Study.J Med Internet Res. 2021;23(1):e23126. doi: $10.2196 / 23126$.

[8] Cao W, Fang Z, Hou G, et al.The psychological impact of the COVID-19 epidemic on college students in China.Psychiatry Res. 2020;287:112934.

doi: 10.1016/j.psychres.2020.112934.

[9] Holman EA, Garfin DR, Silver RC.Media's role in broadcasting acute stress following the Boston Marathon bombings.Proc Natl Acad Sci U S A. 2014 ;111(1):93-8. doi: 10.1073/pnas.1316265110.

[10] Wang C, Pan R, Wan X, et al. Immediate psychological responses and associated factors during the initial stage of the 2019 coronavirus disease (COVID-19) epidemic among the general 
population in China. Int. J. Environ. Res. Public Health. 2020;17(5):1729. doi: 10.3390/ijerph17051729.

[11]Ni MY, Yang L, Leung CMC,et al.Mental Health, Risk Factors, and Social Media Use During the COVID-19 Epidemic and Cordon Sanitaire Among the Community and Health Professionals in Wuhan, China: Cross-Sectional Survey. JMIR Ment Health. 2020 ;7(5):e19009. doi: 10.2196/19009.

[12] Liu S, Yang L, Zhang C, Xiang YT, et al. Online mental health services in China during the COVID-19 outbreak. Lancet Psychiatry. 2020;7(4):e17-e18.

doi: 10.1016/S2215-0366(20)30077-8.

[13] Tunçel KÖ, Selin Ece Taşbakan SE, Deniz Gökengin D,et al.The deep impact of the COVID 19 pandemic on medical students: An online cross - sectional study evaluating Turkish students , anxiety.Int J Clin Pract. 2021; 75(6): e14139. doi: 10.1111/ijcp.14139.

[14]Yin Y, Yang X ,Gao L, et al.The Association Between Social Support, COVID-19 Exposure, and Medical Students' Mental Health. Front Psychiatry. 2021 ;12:555893. doi: 10.3389/fpsyt.2021.555893.

[15] Xiong $\mathrm{P}$, Ming WK, Zhang C, et al.Factors Influencing Mental Health Among Chinese Medical and Non-medical Students in the Early Stage of the COVID-19 Pandemic. Front Public Health. 2021 May 20;9:603331. doi: 10.3389/fpubh.2021.603331. 